\title{
RESONANCE TONGUES IN THE QUASI-PERIODIC HILL-SCHRÖDINGER EQUATION WITH THREE FREQUENCIES
}

\author{
JOAQUIM PUIG AND CARLES SIMÓ
}

\begin{abstract}
In this paper we investigate numerically the following Hill's equation $x^{\prime \prime}+(a+b q(t)) x=0$ where $q(t)=\cos t+\cos \sqrt{2} t+\cos \sqrt{3} t$ is a quasiperiodic forcing with three rationally independent frequencies. It appears,also, as the eigenvalue equation of a Schrödinger operator with quasi-periodic potential.

Massive numerical computations were performed for the rotation number and the Lyapunov exponent in order to detect open and collapsed gaps, resonance tongues. Our results show that the quasi-periodic case with three independent frequencies is very different not only from the periodic analogs, but also from the case of two frequencies. Indeed, for large values of $b$ the spectrum contains open intervals at the bottom. From a dynamical point of view we numerically give evidence of the existence of open intervals of $a$, for large $b$ where the system is nonuniformly hyperbolic: the system does not have an exponential dichotomy but the Lyapunov exponent is positive. In contrast with the region with zero Lyapunov exponents, both the rotation number and the Lyapunov exponent do not seem to have square root behavior at endpoints of gaps. The rate of convergence to the rotation number and the Lyapunov exponent in the nonuniformly hyperbolic case is also seen to be different from the reducible case.
\end{abstract}

"But our tongues get out of control. They are restless and evil, and always spreading deadly poison". (James 3, 6)

To our friend Henk Broer, who likes quasi-periodicity so much, on his 60th birthday.

\section{Introduction}

In this paper we want to study numerically Hill's equation with quasi-periodic forcing, which is the following non-autonomous second order differential equation

$$
x^{\prime \prime}+(a+b q(t)) x=0
$$

Date: July 2, 2010. To appear in Regul. Chaotic Dyn.

2000 Mathematics Subject Classification. Primary: 37B55, 35J10.

Key words and phrases. Quasi-Periodic Schrödinger operators, Quasi-Periodic Cocycles and Skew-products, Spectral Gaps, Resonance Tongues, Rotation number, Lyapunov exponent, Numerical explorations.

The research of J.P. has been supported by grant MTM2009-06973. The research of C.S. has been supported by grant MTM2006-05849/Consolider (Spain). The massive computations have been carried out at the HIDRA cluster of the UB team on Dynamical Systems. 
where $q$, called the forcing, is a quasi-periodic function. This means that it can be written as

$$
q(t)=Q(\omega t+\phi), \quad t \in \mathbb{R}
$$

where $Q: \mathbb{T}^{d}=(\mathbb{R} / 2 \pi \mathbb{Z})^{d} \rightarrow \mathbb{R}$ is a continuous function (although assumed to be real analytic, or even a trigonometric polynomial, in most of what follows), $\phi \in \mathbb{T}^{d}$ is a phase and $\omega=\left(\omega_{1}, \ldots, \omega_{d}\right) \in \mathbb{R}^{d}$ is a frequency vector. We will assume that it is rationally independent in the sense that

$$
\langle\mathbf{k}, \omega\rangle=k_{1} \omega_{1}+\ldots+k_{d} \omega_{d} \neq 0, \quad \mathbf{k} \in \mathbb{R}^{d} \backslash\{\mathbf{0}\} .
$$

Note that the periodic Hill equation occurs when $d=1$ or when all the frequencies are multiple of a single one. Without loss of generality, we will assume that $Q$ has zero average in what follows, changing the value of $a$ if necessary.

A source of interest on these equations is that they are natural generalizations of the classical Hill's equations which appear in the study of the stability of invariant tori, as normal variational equations along a quasi-periodic solution.

Another source of interest is the fact that they show up as eigenvalue equations of one-dimensional Schrödinger operators with quasi-periodic potentials. Let us now briefly recall this setting. A quasi-periodic Hill equation like (1.1), belongs to a family of equations of the form

$$
x^{\prime \prime}+(a+b Q(\omega t+\phi)) x=0,
$$

each of which can be seen as the eigenvalue equation of the following one-dimensional Schrödinger operator with quasi-periodic potential

$$
\left(H_{b, \phi} x\right)=-x^{\prime \prime}-b Q(\omega t+\phi) x,
$$

which is essentially self-adjoint on $L^{2}(\mathbb{R})$. In this context the spectral parameter $a$ is usually called the energy. Contrary to the periodic case, where the spectrum is the union of closed intervals (the spectral bands or the elliptic and parabolic cases for Hill's equation), separated by open intervals in the resolvent set (the spectral gaps or the hyperbolic case for Hill's equation), in the quasi-periodic case the spectrum may be a Cantor set (gaps being dense in the spectrum) and spectral bands may not exist. These operators have been focus of intensive research over the last decades and much is known about the structure of the spectrum of these operators. Note that due to the rational independence of the frequency vector $\omega$, the spectrum of any of these operators, for fixed $b, Q$ and $\omega$ is independent of $\phi$, and will be denoted by $\sigma_{b}(Q, \omega)$ or simply $\sigma_{b}$ if the context is clear.

When the potential $Q$ is real analytic and the frequency satisfies a Diophantine condition, Cantor spectrum has been proved for "small" values of $|b|$, where genericity of Cantor spectrum follows from the genericity of gap opening [33, 18, 7, 36], which also happens generically in the periodic case [32, 9]. As it also happens in the periodic case, one can produce examples of real analytic potentials whose Schrödinger operators have all but a finite number of spectral gaps closed and the spectrum is not a Cantor set [13]. For genericity results in lower regularity, see $[27,19,12,1]$ and references therein.

While the theory for these equations is well understood when $b$ is small or when $a$ is much larger than $b$ (because of the presence of Floquet reducibility, see section 2.1 ), the situation in the other cases is much less clear. In [8] the following example with two Diophantine frequencies was numerically studied,

$$
x^{\prime \prime}+(a+b(\cos t+\cos \gamma t)) x=0,
$$


where $\gamma=(\sqrt{5}-1) / 2$ is the golden mean. For small values of $b$ all the computed spectral gaps were open. When $b$ increases, gaps start to grow until they reach a critical value for which they start shrinking, while they remain open for all computed values. In this present numerical study we want to extend the study of [8] of Equation (1.4) to potentials with three rationally independent frequencies and to try to detect the presence of spectral bands on the bottom of the spectrum for large values of $b$. More precisely we will consider the equation

$$
x^{\prime \prime}+(a+b(\cos t+\cos \sqrt{2} t+\cos \sqrt{3} t)) x=0
$$

and compute the rotation number and the Lyapunov exponent to detect the different characteristics of the Hill-Schrödinger equation in the "perturbative regime" versus the "far from perturbative regime" and the case of 2 versus 3 frequencies. Note that precisely this model was considered as an illustration in [19], where the existence of some gaps was numerically explored.

\section{Preliminaries}

2.1. Dynamical formulation: skew-products flows and reducibility. Many of the recent results and techniques for quasi-periodic Schrödinger operators are based on the spectral properties of (1.3) and the dynamical properties of the eigenvalue equations (1.2). As it is customary for second-order differential equations, they can be written as first order systems with $x^{\prime}=y$ and

$$
\left(\begin{array}{l}
x \\
y
\end{array}\right)^{\prime}=\left(\begin{array}{cc}
0 & 1 \\
-a-b Q(\theta) & 0
\end{array}\right)\left(\begin{array}{l}
x \\
y
\end{array}\right), \quad \theta^{\prime}=\omega
$$

where $\theta=\left(\theta_{1}, \ldots, \theta_{n}\right) \in \mathbb{T}^{d}$ are new angular variables. A system of this kind will be called a linear skew product flow, and shorthanded as $\left(A_{a, b}, \omega\right)$ where

$$
A_{a, b}(\theta)=\left(\begin{array}{cc}
0 & 1 \\
-a-b Q(\theta) & 0
\end{array}\right) .
$$

Note that the flow can be applied both to vectors in $u \in \mathbb{R}^{2}$,

$$
u^{\prime}=A_{a, b}(\theta) u, \quad \theta^{\prime}=\omega
$$

and to matrices in $S L(2, \mathbb{R})$ and this is why we will refer to (2.1) as a skew-product flow on $S L(2, \mathbb{R})$.

As it is well-known, linear periodic systems (e.g. when $d=1$ in (2.1)) are always reducible to constant coefficients by means of a periodic transformation (the socalled Floquet theory). In the quasi-periodic case, such Floquet theory is not always present. Nevertheless, given a linear skew-product flow like (2.1), it is reducible to constant coefficients whenever there is a regular transformation $Z: \mathbb{T}^{d} \rightarrow S L(2, \mathbb{R})$ such that the change of variables

$$
u=Z(\omega t) v
$$

renders the system (2.2) into constant coefficients:

$$
v^{\prime}=B v, \quad \theta^{\prime}=\omega
$$

and $B \in \operatorname{sl}(2, \mathbb{C})$ is a constant matrix, independent of $\theta$, called the Floquet matrix. Note that neither the Floquet matrix nor the reducing transformation, when they exist, are uniquely determined, just as in the periodic case. Sometimes it may also be necessary to halve the frequency, again as in the periodic case. It can be 
seen that such reduction is equivalent to the fulfillment of the following homological equation

$$
\langle\nabla Z, \omega\rangle \equiv \sum_{j=1}^{d} \omega_{j} \partial_{\theta_{j}} Z(\theta)=A(\theta) Z(\theta)-Z(\theta) B, \quad \theta \in \mathbb{T}^{d},
$$

and that the fundamental matrix solution of a reducible skew-product flow can be expressed in terms of the following Floquet representation

$$
X(t)=Z(\omega t+\phi) e^{t B} Z(\phi)^{-1},
$$

where $\theta(0)=\phi$. When a quasi-periodic linear skew-product is a real analytic perturbation of a system with constant coefficients and the frequencies are Diophantine, there is generically reducibility for a "large set" $[5,29]$ or even "full measure" set of perturbations $([18,24])$, using modified KAM schemes, see Section 2.3.

Virtually all dynamical information of a reducible system can be extracted from the properties of a Floquet matrix. For instance, when the Floquet matrix of a reducible system has a negative determinant, then the corresponding skew-product flow has no bounded solution on $\mathbb{R}$ (for any initial phase $\theta$ ), except for the trivial one, so that it has an exponential dichotomy [38] (or that it is uniformly hyperbolic) and all solutions grow exponentially either at $+\infty$ or $-\infty$. It turns out that this concept is intimately related to the spectrum of the corresponding operator (1.3): a Schrödinger operator like (1.3) has a value $a$ in the spectrum if, and only if, the corresponding skew-product flow on $\mathbb{R}^{2} \times \mathbb{T}^{d}$ has an exponentially dichotomy. In other words, if and only if, there is a $\phi \in \mathbb{T}^{d}$ such that equation (1.2) has a nontrivial bounded solution.

In general, to measure the exponential growth of the solutions of a linear skewproduct flow one can use the (upper) Lyapunov exponent,

$$
\beta(a, b)=\lim _{T \rightarrow \infty} \frac{1}{T} \int_{\mathbb{T}} \log \left\|X_{a, b}(t ; \theta)\right\| d \theta,
$$

where $X_{a, b}(\cdot ; \theta)$ is a fundamental solution of the skew-product with $\theta(0)=\theta$. This limit is known to exist by Kingman subadditive ergodic theorem [31]. Note that skew-product flows with an exponential dichotomy have positive Lyapunov exponent if the system is analytic and the frequencies are Diophantine. Skew-product flows on $S L(2, \mathbb{R})$ whose upper Lyapunov exponent is positive but are not uniformly hyperbolic are consistently called nonuniformly hyperbolic, which can never be reducible to constant coefficients.

2.2. Rotation number, spectral gaps and resonance tongues. The relation between exponential dichotomy and the resolvent set (i.e. the complement of the spectrum in $\mathbb{R}$ ) is not the only link between the dynamics of (2.1) and the spectral properties of (1.3). A further insight is obtained by means of the rotation number, introduced by Johnson \& Moser [28] (see also [25] for the discrete version), which measures how solutions wind around the origin. Given a quasi-periodic Hill equation (1.1), its rotation number is given by the limit

$$
\operatorname{rot}(a, b)=\lim _{T \rightarrow+\infty} \frac{\arg \left(x^{\prime}(T)+\mathrm{i} x(T)\right)}{2 \pi T},
$$

where $x$ is any non-trivial solution of (1.1). This number exists and is independent of the chosen solution. It is also independent of the phase taken in (1.2), so that any solution of the skew-product flow (2.1) gives rise to the same limit. The existence 
of this limit for the Hill-Schrödinger equations like (1.2) is related to the fact that dynamics can be transported to the setting of quasi-periodically forced circle flows, since the new angular variable

$$
\varphi(t)=\arg \left(x^{\prime}(t)+\mathrm{i} x(t)\right)
$$

satisfies the following differential equation

$$
\varphi^{\prime}=(a+b Q(\omega t+\phi)) \sin ^{2} \varphi+\cos ^{2} \varphi .
$$

Note that, when $b=0$ then the spectrum is $[0,+\infty)$ and $\operatorname{rot}(a, 0)=\sqrt{a}$ for positive values of $a$ while it is constant and equal to zero on the resolvent set. This is no coincidence since, for any fixed value of $b$, the spectrum is precisely the set of values of $a$ where the function $a \mapsto \operatorname{rot}(a, b)$ (which is a nondecreasing function) is not locally constant [28]. The connected components of the resolvent set (or, equivalently, points of constancy of the rotation number) are therefore the spectral gaps. Moreover, the "Gap Labelling Theorem" [28], states that in any of such gaps, the rotation number must be resonant with respect to $\omega$, that is of the form

$$
\alpha_{\mathbf{k}}=\frac{\langle\mathbf{k}, \omega\rangle}{2},
$$

where $\mathbf{k} \in \mathbb{Z}^{d}$ is a suitable multi-integer such that $\langle\mathbf{k}, \omega\rangle \geq 0$. The minimum value of such resonances, $\mathbf{k}=\mathbf{0}$ corresponds to the lowest gap in the spectrum, which is a semi-infinite interval from $-\infty$ to a certain value of $a$ (called the lowest energy in the spectrum).

Not every gap predicted by the Gap Labelling Theorem is open and we will talk of a collapsed spectral gap with label $\left.\alpha_{\mathbf{k}}=\langle\mathbf{k}, \omega\rangle / 2\right\rangle 0$ whenever, for a fixed value of $b$, the set of energies for which the rotation number equals to $\alpha_{\mathbf{k}}$ reduces to a single point which, therefore, belongs to the spectrum. See Section 2.3 for a discussion of gap opening in the perturbative setting. Nevertheless if all gaps are open then the spectrum is necessarily a Cantor set since it is given by the points of increase of a continuous increasing function which is constant whenever it takes a resonant value and these are dense in $[0,+\infty)$.

For a better understanding of the possible collapse of gaps, it is useful to resort to the concept of resonance tongues, which generalizes the well-known "instability tongues" in the classical Hill periodic equation $[6,9]$. Given any $\mathbf{k} \in \mathbb{Z}^{d}$ such that $\alpha_{\mathbf{k}}=\langle\mathbf{k}, \omega\rangle / 2 \geq 0$, the resonance tongue associated to $\mathbf{k}$ (or equivalently to $\langle\mathbf{k}, \omega\rangle / 2)$ is the set of values in the $(a, b)$-parameter plane whose rotation number equals to $\alpha_{\mathbf{k}}$. The interior of resonance tongues is the union of all the intervals of the resolvent set as a function of $b$.

The resonance tongue with rotation number $\alpha_{\mathbf{k}}=\langle\mathbf{k}, \omega\rangle / 2$ emanates from the point $\left(\alpha_{\mathbf{k}}^{2}, 0\right)$ and, like in the periodic case, its boundaries can meet at a value of $b \neq 0$ (where the corresponding gap is collapsed) yielding what we will call an instability pocket. See $[9,7,36]$ for the analysis in the periodic and quasi-periodic cases, [37] for the discrete case and also the next section.

2.3. The perturbative situation. The structure of resonance tongues and gaps for small values of $|b|$ and for large $a$ (once $b$ has been fixed) can be understood in terms of the reducibility of the corresponding skew-product flows. Although this has been done in detail in [7], let us now give the basic ideas. 
2.3.1. Small $|b|$. As we noted before, resonance tongues emanate from points in the $b=0$ axis of the form

$$
a_{\mathbf{k}}=\alpha_{\mathbf{k}}^{2}=\langle\mathbf{k}, \omega\rangle^{2} / 4, \quad \mathbf{k} \in \mathbb{Z}^{d} \text { with }\langle\mathbf{k}, \omega\rangle \geq 0
$$

and for any such $\mathbf{k} \neq 0$ they correspond to the tips of tongue shaped regions which, for every value of $b$ are given by an interval $\left[a_{\mathbf{k}}^{-}(b), a_{\mathbf{k}}^{+}(b)\right]$ whose interior, if not empty, is an open gap in the spectrum. Let us now consider the structure of the tongue around the tip at $b=0$ using reducibility theory. Note that when $b=0$, the matrix of (2.1) is in constant coefficients with matrix

$$
A_{a_{\mathbf{k}}, 0}=\left(\begin{array}{cc}
0 & 1 \\
-a_{\mathbf{k}} & 0
\end{array}\right) \text {. }
$$

A fundamental solution for $a=a_{\mathbf{k}}$ and $b=0$ is thus given by

$$
X(t)=\left(\begin{array}{rr}
\cos \left(\alpha_{0} t\right) & \alpha_{0}^{-1} \sin \left(\alpha_{0} t\right) \\
-\alpha_{0} \sin \left(\alpha_{0} t\right) & \cos \left(\alpha_{0} t\right)
\end{array}\right)
$$

which is quasi-periodic in $t$ with frequency $\omega / 2$. One can, therefore, consider the change of variables $v=Z_{\mathbf{k}}(\omega t / 2) u$ where

$$
Z_{\mathbf{k}}(\theta)=\left(\begin{array}{rr}
\cos \langle\mathbf{k}, \theta\rangle & \alpha_{0}^{-1} \sin \langle\mathbf{k}, \theta\rangle \\
-\alpha_{0} \sin \langle\mathbf{k}, \theta\rangle & \cos \langle\mathbf{k}, \theta\rangle
\end{array}\right)
$$

which transforms (2.1) into

$$
v^{\prime}=\left(\left(a-a_{\mathbf{k}}\right)+b Q(\theta)\right)\left(\begin{array}{cc}
z_{11} z_{12} & z_{12}^{2} \\
-z_{11}^{2} & -z_{11} z_{12}
\end{array}\right) v,
$$

where

$$
\begin{aligned}
z_{11}^{2}(t) & =\cos ^{2}\left(\alpha_{0} t\right)=\frac{1}{2}+\frac{1}{2} \cos \left(2 \alpha_{0} t\right), \\
z_{12}^{2}(t) & =\frac{1}{a_{0}} \sin ^{2}\left(\alpha_{0} t\right)=\frac{1}{2 a_{0}}-\frac{1}{2 a_{0}} \cos \left(2 \alpha_{0} t\right), \\
z_{11}(t) z_{12}(t) & =\frac{1}{2 \alpha_{0}} \sin \left(2 \alpha_{0} t\right)
\end{aligned}
$$

are quasi-periodic with frequency $\omega$. The transformed system (2.7) is now a perturbation of a constant matrix if we introduce new local coordinates $\delta=a-a_{\mathbf{k}}$ and denote the new skew-product flow by $\left(B_{\delta, b}, \omega\right)$, which satisfies $B_{0,0}=0$. To study this system one can apply any number $r$ of averaging steps [7] to conjugate it analytically to a new skew-product $\left(B^{r}, \omega\right)$ of the form

$$
B^{r}(\theta, \delta, b)=M^{r}(\delta, b)+P^{r+1}(\theta ; a, b)
$$

where the remainder, $P^{r+1}$, which contains the $\theta$-dependence, is of order $r+1$ in $(\delta, b)$ and $M^{r}$ is a matrix independent of $\theta$ with $M^{r}(0,0)=0$. Although this process is not convergent as $r \rightarrow \infty$, the information obtained at any finite cut $r$ is sufficient to obtain the formal Taylor expansions of tongue boundaries up to order $r$ which coincide with the tongues we would obtain if the remainder $P^{r+1}$ was zero (see [7] for a thorough description). For instance, using the form of $B^{1}$ obtained after (2.7), the derivatives of the two tongue boundaries at $b=0$ are given by $\left(a_{ \pm}^{\mathbf{k}}\right)^{\prime}(0)=-Q_{0} \pm Q_{\mathbf{k}}$ where $Q_{\mathbf{k}}$ are the Fourier coefficients of $Q$. In particular, the $\mathbf{k}$-th tongue is transversal at $b=0$ if, and only if, the corresponding harmonic of $Q$, $Q_{\mathbf{k}}$, does not vanish. Although generically this condition of transversality always holds, it may be hard for a concrete potential to detect the order of contact of the 
tongue boundaries at $b=0$. For instance, it was seen in [7] that for Equation (1.4) the order of tangency of tongue boundaries at $b=0$ is exactly $\|\mathbf{k}\|_{1}$ for almost all frequencies, although not for all frequencies (not even Diophantine frequencies).

To transfer this "formal" knowledge at $b=0$ to small values of $|b|$ (and consider for instance the smoothness of tongue boundaries) it is necessary to resort to Eliasson's reducibility theory. In this context, this reducibility theory $[18,22]$ implies that when $\omega \in D C(c, \tau)$ is Diophantine with constants $c$ and $\tau$ and $Q$ is real analytic in some complex strip of width $\rho$, then for sufficiently small $\left|a-a_{\mathbf{k}}\right|$ and $|b|$ the perturbed skew-product flow $\left(B_{\delta, b}, \omega\right)$ is reducible to constant coefficients provided the rotation number is either resonant with respect to $\omega$ or Diophantine with respect to $\omega$. The latter means that the rotation number $\operatorname{rot}(a, b)$ satisfies the estimates

$$
\left|\operatorname{rot}(a, b)-\frac{\langle\mathbf{k}, \omega\rangle}{2}\right| \geq K\|\mathbf{k}\|_{1}^{-\sigma}, \quad \mathbf{k} \in \mathbb{Z}^{d}-0
$$

for some constants $K, \sigma>0$. This reducibility was used in [36] to prove analyticity of tongue boundaries for $|b|$ small, which together with the genericity of transversality of tongue boundaries at $b=0$ gives genericity of Cantor spectrum. Indeed, once a tongue is open for some small value of $b$ it can collapse at a particular value of $b$ but since tongue boundaries are analytic, such tongue tips are separated by intervals of $b$ where the tongue remains open.

In this perturbative context, it can also be shown that both the Lyapunov exponent and the rotation number are Hölder- $\frac{1}{2}[22]$, with square-root behavior at endpoints of open spectral gaps [7] and that the width of gaps decays sub-exponentially according to the label [22].

2.3.2. Large $a, b$ fixed. A second perturbative scenario occurs when $a$ is large and $b$ is fixed because the system is close to a constant rotation with angle $\sqrt{a}$. Indeed, let us take $b$ fixed and consider (2.1) for $a=\alpha^{2}$ and $\alpha \gg 0$. The change of variables

$$
w=\left(\begin{array}{cc}
1 & 0 \\
0 & \alpha
\end{array}\right) y
$$

transforms (2.1) into

$$
w^{\prime}=\left[\left(\begin{array}{rr}
0 & \alpha \\
-\alpha & 0
\end{array}\right)-\frac{b Q(\theta)}{\alpha}\left(\begin{array}{ll}
0 & 0 \\
1 & 0
\end{array}\right)\right] w, \quad \theta^{\prime}=\omega .
$$

If $b / \alpha$ is small, the skew-product flow becomes a small quasi-periodic perturbation of a fast rotation with angular velocity $\alpha$. This approach was taken by Dinaburg \& Sinai [17] and further developed by Eliasson [18] to show that for $a$ large enough, the skew-product is reducible when the rotation number is either resonant or Diophantine with respect to $\omega$. The averaging approach for this fast periodic forcing [39] works very well and one can obtain the asymptotics of the rotation number and the Lyapunov exponent at $+\infty[16]$ together with an estimate on the decay of gap length [15]. In this real analytic setting, the rotation number behaves as $\sqrt{a}$ at $+\infty$ and gap length decays exponentially.

The generic opening for small $b$ is also transferred to this situation since there is a global Lipschitz bound on tongue boundaries [7] which implies that for $B$ fixed and sufficiently large $A_{B}$ the skew-product flow at the boundaries of any tongue in $a>A_{B}$ and $|b|<B$ is reducible to constant coefficients and therefore has real analytic boundaries. 
Both the situation for small $|b|$ in Section 2.3.1 and large $a$ and $b$ fixed in Section 2.3.2 will be referred as the reducible domain where, according to Eliasson reducibility theory, skew-products whose rotation number is either Diophantine or resonant with respect to $\omega$ are reducible to constant coefficients and, generically the spectrum is a Cantor set with (again generically) all gaps open $[18,36]$. It is important to stress that this does not imply the reducibility for all values of $(a, b)$ in this domain [18] even if the Lyapunov exponent is zero on the domain. In fact, as proved in [35], any subset of the spectrum with zero Lyapunov exponent which is a Cantor set contains a $G_{\delta}$ subset of energies which are not reducible to constant coefficients. In the reducible domain, however, this set of nonreducible energies has always zero Lebesgue measure [18].

2.4. Outside the reducible domain. Even if in the reducible domain there are generically values of $a$ and $b$ whose linear skew-product flows are not reducible to constant coefficients, their Lebesgue measure is zero. Nevertheless, for a fixed real analytic $Q$, Diophantine frequencies and $b$ sufficiently large, there might be a region at the bottom of the spectrum where the Lyapunov exponent is positive. Hence the corresponding skew-products are nonuniformly hyperbolic and, thus, nonreducible to constant coefficients. Inside the gaps in this region, though, the system is reducible to constant coefficients (if $Q$ is analytic and the frequencies are Diophantine).

The first results in this direction were considered by Frölich et al. [20] in the case of the potential with two frequencies given by (1.4) when $b$ is large and $\gamma$ is any Diophantine frequency. More precisely they show that if $b$ is sufficiently large, there is an interval with a nonvoid intersection with the spectrum where the spectrum is pure-point with exponentially localized eigenfunctions for almost every phase. The existence of such eigenfunctions for almost all phases, which are solutions of the eigenvalue equation (1.2) decaying exponentially at $-\infty$ and $+\infty$ and hence belonging to $L^{2}(\mathbb{R})$, is referred as Anderson localization and implies positivity of the Lyapunov exponent.

The positivity of the Lyapunov exponent at the bottom of the spectrum was generalized by Bjerklöv [3] who considered real analytic potentials $Q: \mathbb{T}^{d} \rightarrow \mathbb{R}$ and Diophantine frequencies. For sufficiently large $b$ it was shown that on the interval $\left[0, b^{\frac{2}{3}}\right]$ the Lyapunov exponent is greater than $c_{0} \sqrt{b}$ where $c_{0}$ is a positive constant depending only on $Q$ and this interval contains energies in the spectrum, provided that $b$ is sufficiently large.

The picture for the Lyapunov exponent is thus the following in this real analytic context: for small values of $b$ the whole spectrum is included in the reducible domain but for a critical value of $b$, the Lyapunov exponent for the lowest energy in the spectrum (which is the largest energy $a$ with zero rotation number) becomes positive. As $b$ grows, bigger portions at the bottom of the spectrum turn to have positive Lyapunov exponent whereas larger energies $a$ are in the reducible domain (see Section 2.3.2). For large $b$, thus, the system undergoes a dramatic transition when $a$ goes to infinity. Previous numerical work on the model (1.4) indicates that there is a critical curve in the $(a, b)$-plane where the Lyapunov exponent becomes positive. Interestingly in [8] it was observed that nearby resonance tongues seem to cluster around when they cross this curve and that the set of values on the critical curve which are outside resonance tongues has zero Lebesgue measure, see Section 4 and also the discrete case in [37]. 
Very little is known about the structure of the spectrum in the region of positive Lyapunov exponents. A little bit more is known for discrete Schrödinger operators on $l^{2}(\mathbb{Z})$,

$$
\left(H_{b V, \phi} x\right)_{n}=x_{n+1}+x_{n-1}+b V(\omega n+\phi) x_{n}, \quad n \in \mathbb{Z},
$$

where $V: \mathbb{T}^{d} \rightarrow \mathbb{R}$ is a potential, $d \geq 1$ and $\omega=\left(\omega_{1}, \ldots, \omega_{d}\right) \in \mathbb{R}^{d}$ is an irrational frequency vector in the sense that

$$
\langle\mathbf{k}, \omega\rangle=k_{1} \omega_{1}+\ldots+k_{d} \omega_{d} \notin 2 \pi \mathbb{Z} \text { for all } \mathbf{k}=\left(k_{1}, \ldots, k_{d}\right) \in \mathbb{Z}^{d} \backslash\{\mathbf{0}\} .
$$

Most of the definitions given for the continuous case also apply in this discrete context. A difference with the continuous case, however, is that here the spectrum is always a compact subset of $\mathbb{R}$ and if $V$ is real analytic and the frequencies Diophantine, the Lyapunov exponent is always positive on the whole spectrum for sufficiently large $b$ (see the review and references in [26]).

In the discrete case, when $b$ is large and there is a single Diophantine frequency, the spectrum is a Cantor set $[41,21,23]$ although it was numerically observed in [37] that when the potential has several maxima and minima a number of gaps can collapse in a non-smooth way in this domain.

The situation in the discrete case with two or more frequencies (which corresponds to the continuous case with three or more frequencies) is very different in the regime of positive Lyapunov exponents, as indicated by work by Chulaevsky and Sinai [10]. More precisely, for $|b|$ large enough and "non-degenerate" potentials the spectrum might consist of a single spectral band without any gap. This phenomenon of gap closing was numerically studied in [37] for several examples, as it will be done for the continuous case in Section 4 .

\section{NumericAl METHODS}

To compute numerically both Lyapunov exponents and rotation numbers we must integrate $x^{\prime \prime}+p(t) x=0$ or, as a system,

$$
x^{\prime}=-y, \quad y^{\prime}=p(t) x,
$$

where $p(t)=a+q(t)$. It is quite convenient to use Taylor methods (see, e.g. [30] and references therein, and also the lecture notes in [40] which discuss when the method is suitable and display several examples). As $q(t)=\cos \left(\theta_{1}\right)+\cos \left(\theta_{2}\right)+\cos \left(\theta_{3}\right)$, where $\theta_{j}=\omega_{j} t$, the jet to any order of $p(t)$ contains coefficients $b \omega_{j}^{k} / k$ ! which can be precomputed for fixed $b$. The computational cost per step using order $N$ is of the form $a_{0}+a_{1} N+N^{2}$, measured in elementary operations $\left(+,{ }^{*}\right)$, in the present case, and the first two terms can not be neglected in front of $N^{2}$. This implies that the optimal order is large. Setting the local truncation error to $10^{-21}$, so that it can be neglected in front of the roundoff errors, the optimal order has been found to be near $N=40$ and this value has been used for all the computations, carried out in double precision.

Different computers have been used, but to have a performance idea, running under Linux on a laptop at $1.86 \mathrm{GHz}$ produces 200,000 integration steps per second, with an average step size near $\pi / 4$, in the explored domain.

As an additional check the fundamental matrix $X(t)$ associated to (3.1) with $X(0)=I$ has been computed for values of $(a, b)$ with zero Lyapunov exponent, like $(4,1)$. The values of $\operatorname{det}(X)-1$ can be used to check the accuracy of the solutions. 


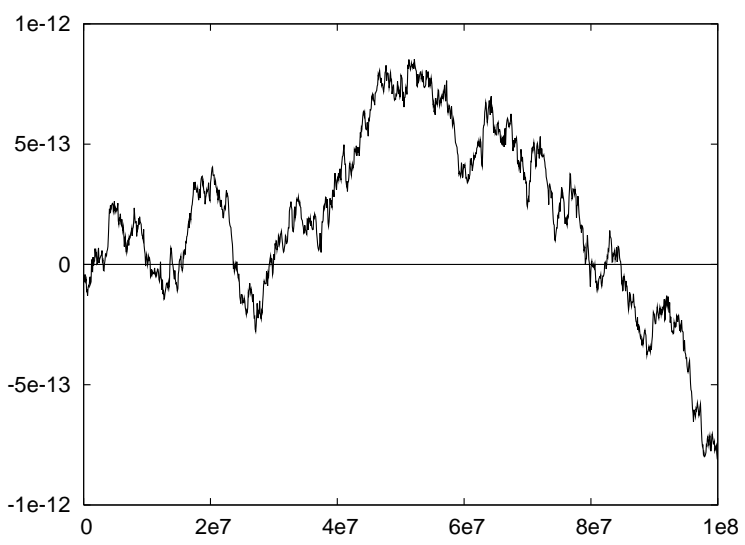

FiguRE 1. Errors in $\operatorname{det}(X(t))-1$, where $X$ is a fundamental matrix of the Equation (1.1) with $X(0)=I$. Time is displayed on the horizontal axis. In this example the values $(a, b)=(4,1)$ have been used.

A typical random walk behavior is obtained, as can be seen in Figure 1. After this check we only integrate (3.1).

As $\omega_{1}=1$ we can consider the discrete map associated to one revolution of $\theta_{1}$, i.e. passages of $\theta_{1}$ through multiples of $2 \pi$. For the subsequent computations the Lyapunov exponent has been computed taking $2 \pi$ as unit of time. Let a variable $L$ be initially set to zero. Starting with the initial vector $(1,0)^{T}$ let $v(1)$ be the vector obtained after one revolution. Then $L$ is updated by adding to it $\log \left(|v(1)|_{2}\right), v$ is normalized by replacing it by $v(1) /|v(1)|_{2}$ and the computation is continued. Let us denote as Lyapunov sums $L_{n}$ the values of $L$ as a function of the number of revolutions $n$. The Lyapunov exponent can be computed as the slope of a linear fit of the values of $L_{n}$ as a function of $n$ up to a maximal value $N_{\max }$.

In fact, both to reduce the size of the sample and to smoothen a little the behavior of $L_{n}$, one keeps only for the fit values of $n$ for which the variables $\theta_{2}, \theta_{3}$ are close to zero modulus $2 \pi$. This criterion is close to the "right stop criterion" used in [11] to compute Lyapunov exponents in a general setting, and interesting characteristics will be commented later.

Concerning the rotation number, one can proceed in a similar way by doing fits of $S_{n}$ as a linear function of $n$, where $S_{n}$ is half the number of crossings of the $y$ component of the vector through $y=0$ in the positive sense, which is equivalent to the definition given in Section 2.2. Note that as $p(t)$ can be negative, changes of sign in the reverse sense can occur.

To illustrate the difficulties associated to the lack of reducibility, Figure 2 shows the oscillations of $L_{n}$ and $S_{n}$ with respect to the fits in a moderate domain of $n$. The figure suggests that one has to use large values of $N$ to obtain accurate estimates of the Lyapunov exponent and the rotation number. Typical values of $N_{\max }$ in the computations range in $\left[10^{5}, 10^{6}\right]$. For some details larger values, even exceeding $10^{9}$, have been used. The computed values are very accurate in the reducible domains. Hence, to avoid exceedingly long computations, for a sequence of increasing values of $N$ (say, of the form $N=k \times N^{*}, k \geq 5$ ) we have computed the slopes of $L_{n}$ and $S_{n}$ using the last $60 \%, 40 \%$ and $20 \%$ of the data. Let $s_{60}^{L}, s_{40}^{L}, s_{20}^{L}$ and $s_{60}^{S}, s_{40}^{S}, s_{20}^{S}$ 
be the values obtained. As done in [37] the computation has been stopped if

$$
\max \left\{\left|s_{60}^{L}-s_{40}^{L}\right|,\left|s_{40}^{L}-s_{20}^{L}\right|\right\} \quad \text { and } \max \left\{\left|s_{60}^{S}-s_{40}^{S}\right|,\left|s_{40}^{S}-s_{20}^{S}\right|\right\}
$$

are less than some prescribed tolerance, typically taken as $1 / N_{\max }$. Then the values $s_{40}^{L}$ and $s_{40}^{S}$ are used as suitable estimates. Otherwise the computation continues until $N=N_{\max }$.
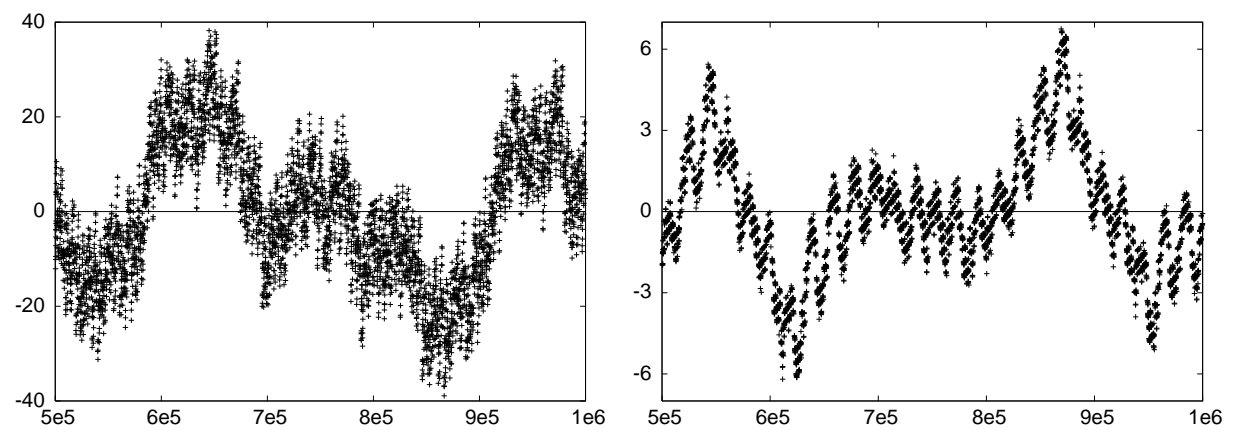

Figure 2. Evolution of the Lyapunov sums $L_{n}$ (left) and of half the number of changes of sign of $y, S_{n}$, (right) having subtracted the linear fit, as a function of the number of revolutions $n$ of $\theta_{1}$, in the interval $\left[5 \times 10^{5}, 10^{6}\right]$. The values $(a, b)=(0,1)$ have been used.

In the figures displayed in Section 4 one has used a step in $a$, along lines of constant $b$, equal to 0.005 for the larger domains (Figure 5). The step is reduced to $10^{-3}, 10^{-5}$ and $10^{-4}$ for details in Figures 6,7 and 8 , respectively. The zones with resonant rotation number or the ones with zero Lyapunov exponent (e.g., in Figures 5 to 8) display the pixels for which the related condition is satisfied. When from one pixel to next one a resonance is crossed the corresponding value of $a$ is obtained by interpolation, but keeping only resonances up to order $\mathrm{m},|\mathbf{k}|_{1} \leq m$, for different values of $m$, to avoid having too many lines.

\section{Results}

For the numerical exploration we considered equation (1.5),

$$
x^{\prime \prime}+(a+b(\cos t+\cos \sqrt{2} t+\cos \sqrt{3} t)) x=0,
$$

whose frequency vector $\omega=(1, \sqrt{2}, \sqrt{3})$ is Diophantine. We computed numerically the rotation number and the Lyapunov exponent for large sets of $(a, b)$ values. See Figures 3 and 4 for preliminary illustrations.

The two regimes which were described in sections 2.3 and 2.4 are detected in terms of the Lyapunov exponent and the rotation number. Indeed, when the Lyapunov exponent is positive outside the resonance tongues, the system is nonuniformly hyperbolic and there is no reducibility to constant coefficients. This is clearly seen in Figure 5 bottom which displays the subset in the $(a, b)$-parameter plane where the Lyapunov exponent is zero and the rotation number is not resonant with respect to $\omega$ (see the caption for more details). This domain contains the whole spectrum for small values of $b$ and a subset for large $a$ once $b$ has been fixed. It is reasonable to believe that the reducible domain (i.e. where skew-products are 


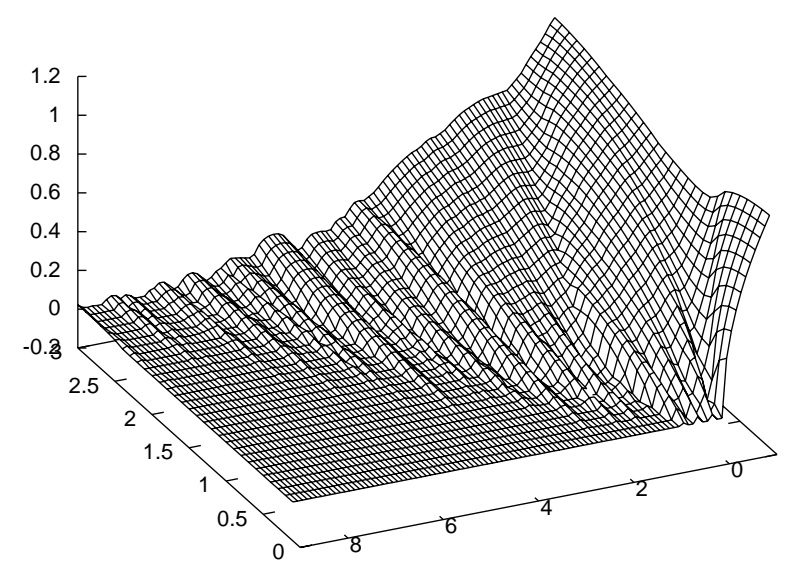

Figure 3. An illustration of the behavior of the Lyapunov exponent, computed as a time average, for $a \in[-1,9], b \in[0.1,4]$. Final time $10^{6}$.

reducible to constant coefficients for almost all rotation number) is given by this shaded region where the Lyapunov exponent is zero. This belief is consistent with results in the discrete case [2] and its validation would imply that, in this region, the skew-products are reducible for Lebesgue almost every value of $a$, while keeping $b$ fixed $[14,2]$.

In the regime of zero Lyapunov exponents (which includes the whole spectrum for $b$ less than 0.183 , see the tip of the leftmost zero-Lyapunov domain in Figure 5 bottom), everything is as predicted by the perturbative theory of Section 2.3:

- The Lyapunov exponent and the rotation number are Hölder- $\frac{1}{2}$ and have square-root behavior at the endpoints of open gaps (see the right-most part of Figure 4 for $b=0.5$ ).

- The order of contact of the $\mathbf{k}$-th tongue at $b=0$ for this particular equation is exactly $\|\mathbf{k}\|_{1}$ and, in particular, only the tongues with rotation number $1 / 2, \sqrt{2} / 2$ and $\sqrt{3} / 2$, which emanate from $a=1 / 4,1 / 2$ and $3 / 4$ are transversal at $b=0$. In [7] it was shown that, in the present situation of an equation like (1.5), this order is at least $\|\mathbf{k}\|_{1}$ and that for almost all frequencies it is exactly this value. When the Lyapunov exponent at tongue boundaries is zero, these boundaries look very smooth, which is consistent with the fact that they are real analytic functions of $b$ whenever the skew-product is reducible at a tongue boundary [36].

- All computed tongues open at $b=0$ (with different orders according to their label) and they do not show any instability pocket if the Lyapunov exponent is zero at the boundaries of the tongue (inside it will always be positive due to the presence of an exponential dichotomy). In particular, they remain open for all small values of $b$ and the spectrum is a Cantor set with all spectral gaps open. The same happens for large values of $a$ and $b$ fixed. Note that the absence of instability pockets in the reducible domain is a very particular feature of this model since they are produced by a generic perturbation [7]. 

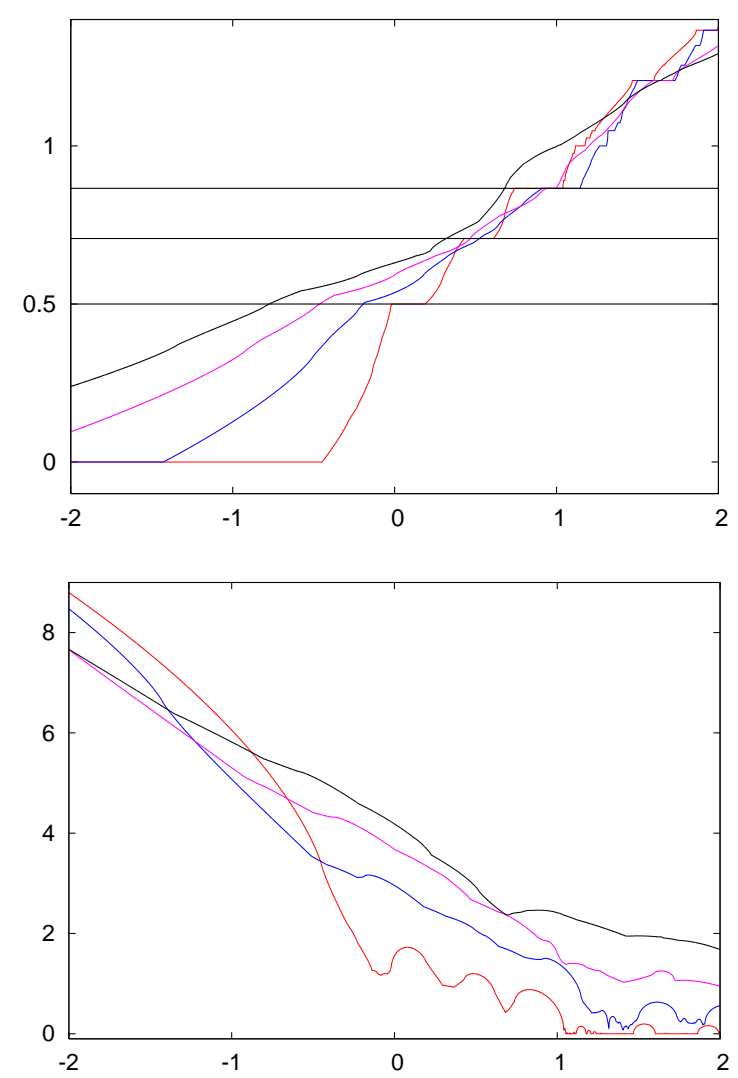

Figure 4. Top: Values of the rotation number for $a \in[-2,2]$. The four curves correspond to values of $b$ equal to $0.5,1.0,1.5,2.0$. For $a=-1$, for instance, the curves are ordered as the values of $b$. The three horizontal lines correspond to rotation numbers $1 / 2, \sqrt{2} / 2$ and $\sqrt{3} / 2$. The bottom plot displays the results for the Lyapunov exponent, as fitted slope with respect to the number of revolutions of $\theta_{1}$. For $a=0.5$ the curves are ordered as the values of $b$.

As seen in Figure 5 when $b$ grows (when it crosses the value $b \approx 0.183$ ) the Lyapunov exponent becomes positive in a compact subset at the bottom of the the spectrum. We can speak of a "critical line" which starts at the bottom of the spectrum when $b \approx 0.183$ and divides the parameter space into the nonuniformly hyperbolic region (at the left-hand side or at the top of the curve) and the reducible domain at the right (or at the bottom). This curve was already numerically observed in the model (1.4) with two frequencies [8] and plays the same role as the line $b=2$ in the Almost Mathieu case, which is the discrete model (2.8) obtained when $V(\theta)=\cos \theta$. Evidences of that critical line can be seen also in the magnifications in Figure 6 and in the highly detailed Figure 8 (where we use $9000 \times 1500$ pixels). Another common feature of our present model with the former is that along this critical line the width of resonance tongues seems to attain its maximum value and 

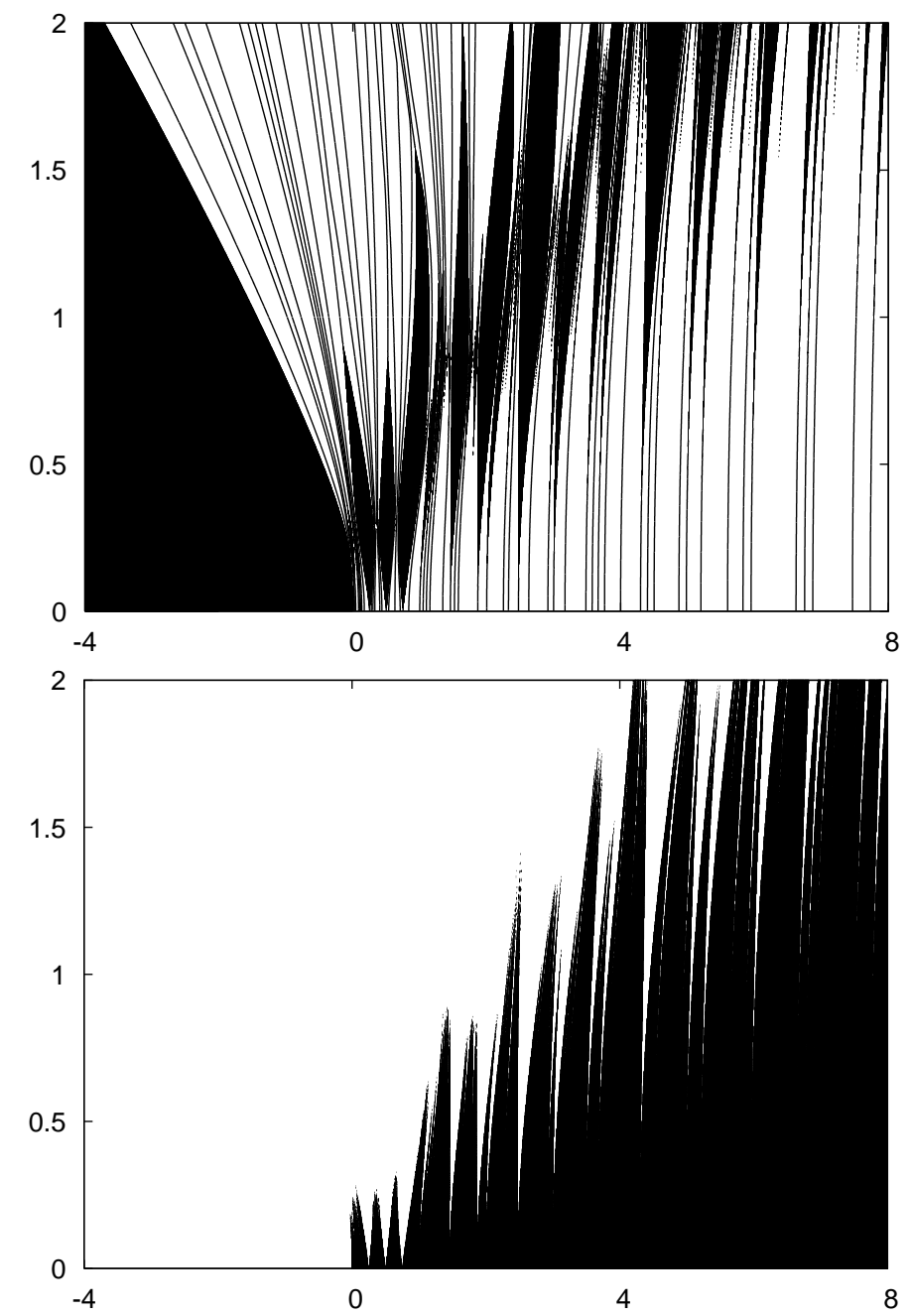

Figure 5. Top: Values of $(a, b)$ for which the rotation number is resonant, see text for details. Only lines with resonances up to order 4 outside the wide resonant zones are displayed. Bottom: Values of $(a, b)$ for which the Lyapunov exponent is zero.

nearby resonance tongues cluster so that the measure of values $(a, b)$ along this curve which lie outside tongues seems to be zero.

In the nonuniformly hyperbolic domain, the situation is substantially different from that of two frequencies. Indeed, in the case of two frequencies of (1.4) the numerical exploration of [8] showed that tongues start shrinking after they cross the critical curve, but they remain always open for larger values of $b$ in the explored domain. In the present case with three frequencies, Figure 5 shows that tongues do shrink when they cross the critical curve but at some height of $b$ they have a pocket which does not reopen after the tip. This can already be guessed in Figure 4 where the resonances associated to rotation numbers $1 / 2, \sqrt{2} / 2$ and $\sqrt{3} / 2$ are related to plateaux for $b=0.5$. The first two have disappeared already for $b=1$ 


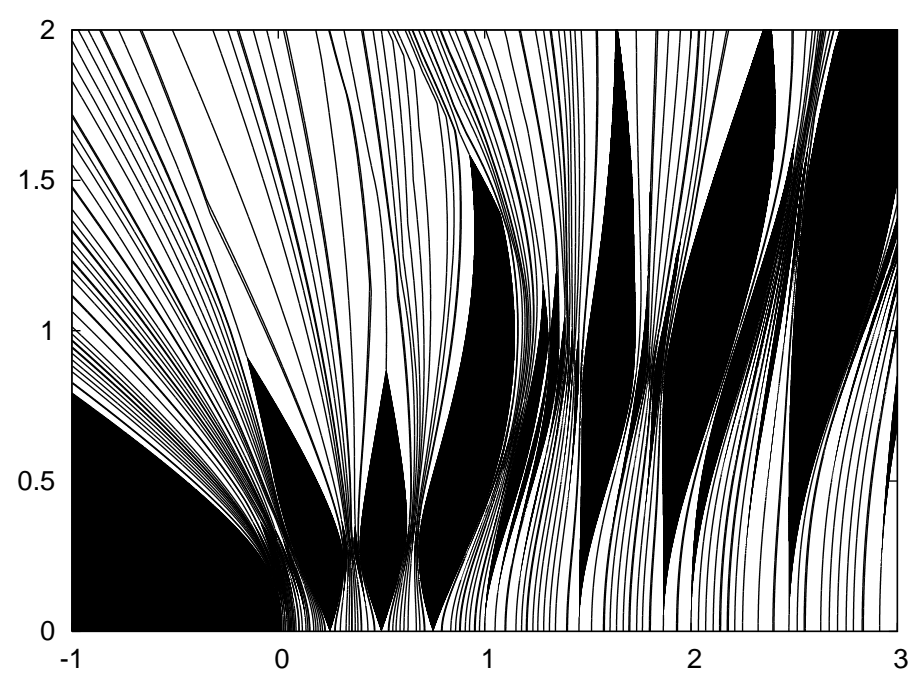

FiguRE 6. Magnification of Figure 5 top. Here we include resonances up to order 8 in the displayed domain.
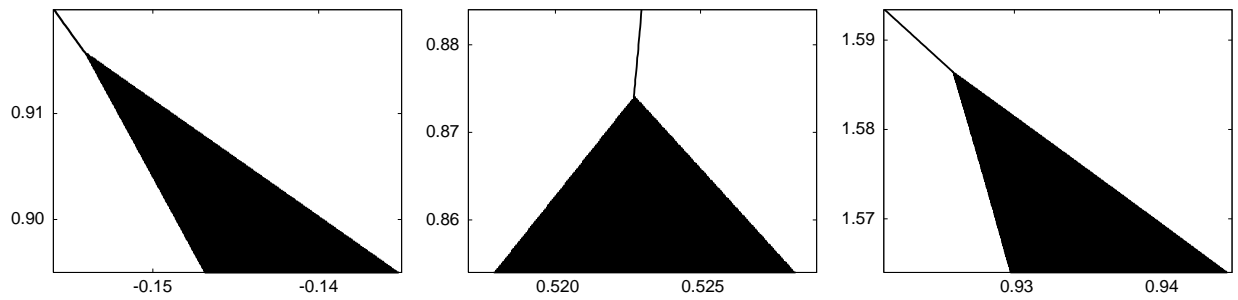

FiguRE 7. From left to right: magnifications of the end tips of the resonances with $\mathbf{k}$ equal to $(1,0,0),(0,1,0)$ and $(0,0,1)$, respectively. They can be seen as the first three large resonant domains in Figure 5, letting aside the $\mathbf{k}=\mathbf{0}$ resonant domain. After the tip only one value of $a$ has been found to have the resonant rotation number for each $b$.

and the last one has disappeared for $b=2$. It is also clearly seen in Figure 6 and in the magnifications of Figure 7 which display the tip for these tongues. After the tongue closes it does not reopen again and larger values of $b$ have a collapsed gap corresponding to that rotation number. This phenomenon will be denoted as persistent gap closing as it is different from the type of gap closing, not persistent, that occurs in the reducible domain. At the point of collapse of a resonance tongue, gap length tends to zero linearly.

As a consequence, as $b$ grows, the bottom of the spectrum contains a spectral band, to the left of a given value of $a$ depending on $b$, which is very different from the spectral bands that appear in the periodic Hill equation. Because the topmost part of the spectrum lies in the reducible domain and there is Cantor spectrum there, we face an example of an spectrum with coexisting spectral bands and portions with Cantor spectrum. 
Once a tongue crosses to the nonuniformly hyperbolic domain, its boundaries may not be smooth. A clear example occurs at the magnifications around the persistent gap closing in Figure 7, where the curve with fixed rotation number that survives in the $(a, b)$-plane after the collapse does not seem to have the direction of any of the two boundaries at the point of collapse, neither the average of the two slopes. Before the collapse, where the tongue is still open, there can also be values of $b$ where a boundary is not smooth. This can be seen in Figure 6 where the tongue with rotation number $\sqrt{3} / 2$ has a singularity of this kind for $b$ around 1.4 at the right endpoint. After the tongue has collapsed, the surviving curve may also have points where it is not smooth. This lack of smoothness was also observed for the discrete models in [37] with two and more frequencies (which correspond to continuous models with three and more frequencies). Figure 8 shows a further magnification of Figure 6 where one can see a better detail of the critical line and several addicional collapsed tongues for small values of $b .^{1}$

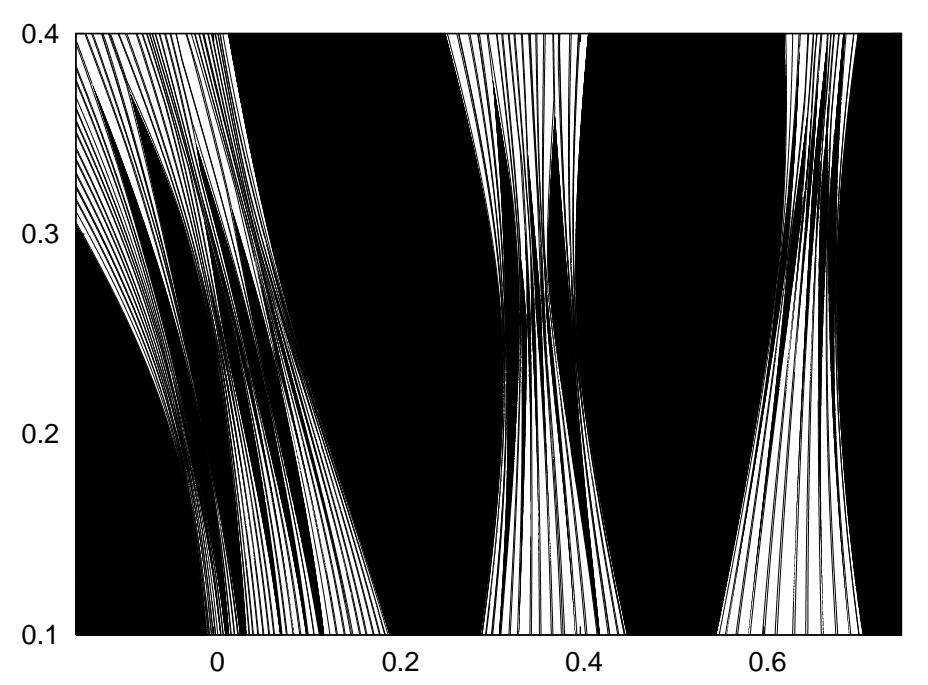

Figure 8. Magnification of Figure 6 using steps $10^{-4}, 2 \times 10^{-4}$ in $a, b$. Here we include lines with resonances up to order 12 in the displayed domain. In the wider resonant zones the highest order detected is 76 .

Concerning the behavior of the rotation number and the Lyapunov exponent in the nonuniformly hyperbolic domain, both the rotation number and the Lyapunov exponent seem to have one-sided derivatives at the endpoint of the surviving open spectral gaps, see Figure 4, contrary to the observed square-root behavior in the reducible domain. Moreover, inside a spectral band with positive Lyapunov exponent, the rotation number seems to have points with different derivatives from the left and the right.

The behavior of the Lyapunov sums $L_{n}$ and the rotation sums $S_{n}$, as introduced in Section 3 is strongly dependent on the reducibility of the system. When the skew-product is reducible to constant coefficients, the Floquet representation $(2.3)$ implies that the differences between $S_{n}$ and $n$ times the final rotation number will

\footnotetext{
${ }^{1}$ Contact the authors for even larger magnifications with steps $10^{-5}, 10^{-4}$, or smaller, in $a, b$.
} 

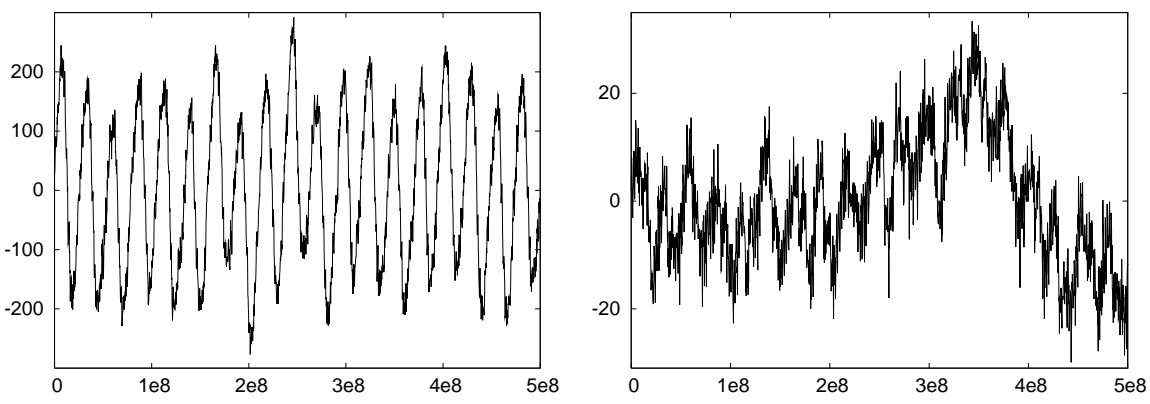

FiguRE 9. Plots like the ones displayed in Figure 2 on the interval $\left[0,5 \times 10^{8}\right]$.
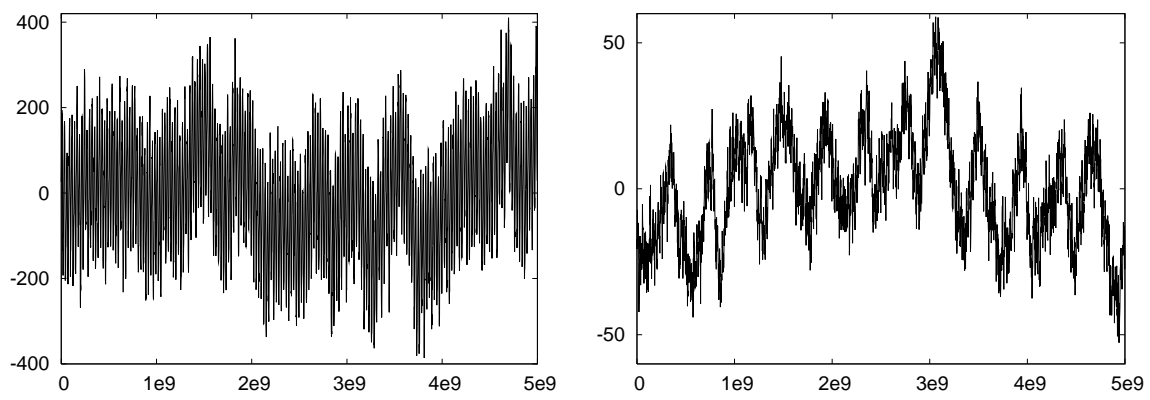

Figure 10. Plots like the ones displayed in Figure 2 on the interval $\left[0,5 \times 10^{9}\right]$.

be bounded and the same happens with the Lyapunov exponent. In the nonuniformly hyperbolic domain, where there is no reducibility to constant coefficients, the differences need not to be bounded, see Figure 12 and related comments. This seems to be related to the concept of $\rho$-boundness for quasi-periodically forced circle maps [4].

In Figure 9, for a final time $10^{9}$, we display the differences between the sums and the linear fits as a function of the number of revolutions of $\theta_{1}$. We see that the oscillations are wild, but one can distinguish many peaks, more clearly in the case of $L_{n}$ in this figure. Figure 10 shows that both pictures look more similar if the final time goes to $5 \times 10^{9}$. We note that, roughly, only one point every $10^{5}$ points is shown. It is interesting to try to identify the reason of the peaks. To this end one has selected different minor domains, giving slightly different estimates of the slopes, and giving evidence of peaks with different spacings.

It seems natural to check for the "quasi-periods" of the function $Q$. Given $\varepsilon>0$ we can look for values of $k \in \mathbb{N}$ such that $Q$ is close to be periodic with period $2 \pi k$. As we consider revolutions when $\theta_{1}=0 \bmod 2 \pi$, it is enough to look for the values of $\theta_{2}, \theta_{3}$ after every revolution of $\theta_{1}$. To this end we can select values of $k$ such that the distance of $k\left(\omega_{2}, \omega_{3}\right)$ to $\mathbb{Z}^{2}$, measured by the Euclidean norm $d_{k}$ is smaller than the given $\varepsilon$. The "best" values are obtained for the $k$ such that $d_{k}<\min \left\{d_{j}, 0<j<k\right\}$. This is related to the problem of simultaneous Diophantine approximation (SDA). Table 1 gives some of the values of $k$ for which this condition is satisfied, as well as the related value of $d_{k}$. Figure 11 shows the 


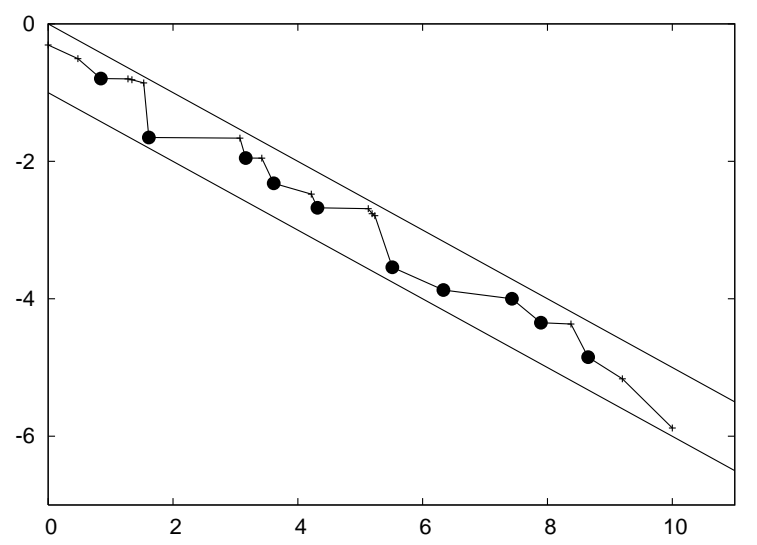

Figure 11. The values of $d_{k}$ for the values of $k$ as defined in Table 1 for all $k<10^{11}$. The large dots correspond to quasi-periods detected in Figures 2, 9, 10 and similar ones (not displayed in the paper) at different scales. The values on the axes correspond to $\log _{10}(k)$ and $\log _{10}\left(d_{k}\right)$.

values of $k \leq 10^{12}$ for which this condition holds. In the figure, $\log _{10}$ scales are used in both axes and the two straight lines are $-x / 2$ and $-x / 2-1$, where $x$ denotes here the horizontal variable. The general trend is a decrease of the form $d_{k}=\mathcal{O}\left(k^{-1 / 2}\right)$, in agreement with classical results on SDA (see, e.g., [34]).

\begin{tabular}{|r|l||r|r|}
\hline 7 & $0.1598924517345656 \mathrm{E}$ & 326491 & $0.2879886604030898 \mathrm{E}-3$ \\
41 & $0.2226404176161304 \mathrm{E}-1$ & 2151016 & $0.1340612832092896 \mathrm{E}-3$ \\
1463 & $0.1115233301766352 \mathrm{E}-1$ & 26963149 & $0.9994228251808058 \mathrm{E}-4$ \\
4109 & $0.4784264961464025 \mathrm{E}-2$ & 78411940 & $0.4480247193528474 \mathrm{E}-4$ \\
20586 & $0.2112644427660709 \mathrm{E}-2$ & 447810523 & $0.1417064795288236 \mathrm{E}-4$ \\
\hline
\end{tabular}

TABLE 1. Euclidean norm $d_{k}$ of the distance of $\left(k \omega_{2}, k \omega_{3}\right)$ to $\mathbb{Z}^{2}$ for some of the values of $k$ for which $d_{k}<\min \left\{d_{j}, 0<j<k\right\}$. Left columns: values of $k$; right columns: values of $d_{k}$. The values shown here are the ones which appear as large dots in Figure 11.

It turns out that some of the values of $k$ correspond to the distances between peaks seen in plots like the ones displayed in Figures 2, 9, 10 and similar ones (not displayed for shortness). These values appear in Figure 11 as large dots.

Finally one can turn the attention to the behavior of the maximal values of the differences between $L_{n}$ and the linear fit, as a function of the maximal value $N_{\max }$ of $n$ which is used in the computations. A similar question can be posed for $S_{n}$. As it is expected these differences stabilise in the reducible case as mentioned before. As a matter of fact, for a number of tests done up to $N_{\max }=10^{9}$, they stabilise at small values. The behavior is quite different in the nonreducible case.

Figure 12 displays these differences for the Lyapunov sums, several values of $N_{\max }$ and values of $a$ equal to $0,0,0.5$ and 1.5 while keeping $b=1$ fixed $(a=1, a=2$ show a reducible character for the same value of $b$ ). The patterns are quite similar in the 


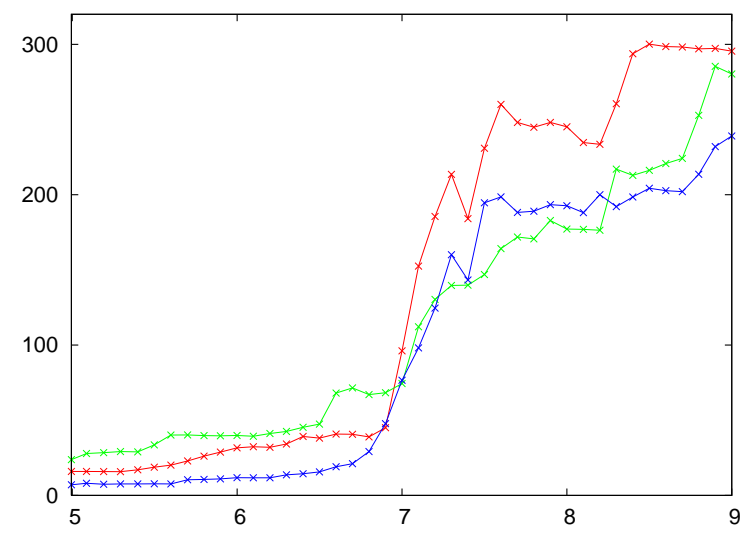

Figure 12. Maximal absolute value of the differences between the Lyapunov sums $L_{n}$ and the linear fits, as a function of the number of revolutions of $\theta_{1}$, up to a maximum of $N$ revolutions for different values of $N$, of the form $N=E\left(10^{5} \times 10^{j / 10}\right)$ for $j=0, \ldots, 40$. In the horizontal axis we display $\log _{10}(N)$. For $b=1$ the red, green and blue curves correspond to $a=0.0,0.5$ and 1.5 , respectively.

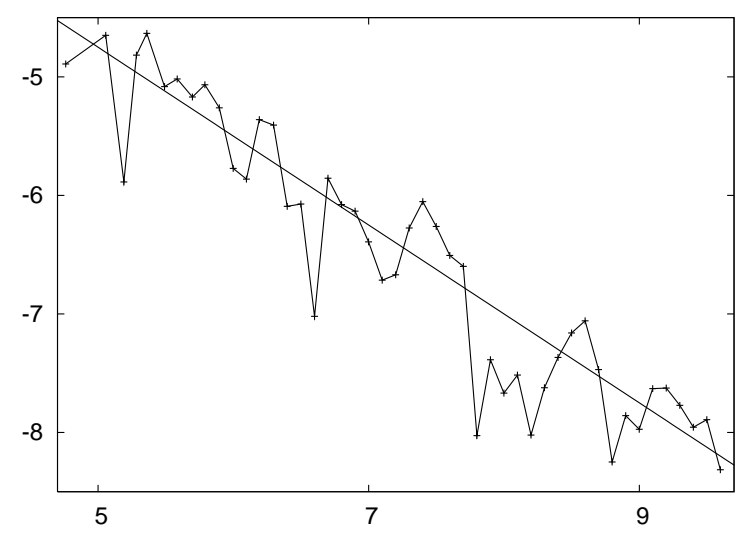

FigURE 13. Let $\rho_{N}$ be the value of the rotation number estimated as the slope of a linear fit of $S_{n}, n \leq N$ (see Figure 2). The plot shows $\log _{10}\left|\rho_{N}-\rho_{N_{0}}\right|$ as a function of $\log _{10}(N)$ for different values of $N$ when one takes $N_{0}=5 \times 10^{9}$. For that computation the values $(a, b)=(0,1)$ have been used. The blue curve has slope $-3 / 4$, very close to the slope of a linear fit of these data.

three cases. The same occurs if $L_{n}$ are replaced by $S_{n}$ showing an increasing trend with sudden jumps at slightly different places. Jumps that seem to be related to the values of $k$ are shown in Table 1 . The range of $N_{\max }$, even reaching $10^{9}$, seems still too short to draw conclusions about the rate of increase. In an alternative way we have proceeded as follows. Let $\rho_{N}$ be the value of the rotation number estimated as the slope of a linear fit of $S_{n}, n \leq N$. Let us take as "more correct estimate" $\rho_{N_{0}}$ the value obtained for $N_{0}=5 \times 10^{9}$. Figure 13 shows the behavior of $\log _{10}\left|\rho_{N}-\rho_{N_{0}}\right|$ as a function of $\log _{10} N$. As one can expect larger values of $N$ 
produce values closer to $\rho_{N_{0}}$, despite large irregularities showing up. A linear fit of these data gives a slope -0.752 and the line drawn on the plot has slope -0.75 . One could be tempted to take the value -0.75 as the correct one, but many longer explorations have to be done before drawing conclusions.

\section{ACKNOWLEDGMENTS}

The research of J.P. has been supported by the MCyT-FEDER Grant MTM200906973 (Spain). C.S. has been supported by grants MTM2006-05849/Consolider (Spain) and CIRIT 2008 SGR-67 (Catalonia).

\section{REFERENCES}

[1] A. Avila, J. Bochi, and D. Damanik. Cantor spectrum for Schrödinger operators with potentials arising from generalized skew-shifts. Duke Math. J., 146(2):253-280, 2009.

[2] A. Avila and R. Krikorian. Reducibility or non-uniform hyperbolicity for quasiperiodic Schrödinger cocycles. Ann. of Math., 164(3):249-294, 2006.

[3] K. Bjerklöv. Positive Lyapunov exponents for continuous quasiperiodic Schrödinger equations. Journal of Mathematical Physics, 47:022702, 4pp, 2006.

[4] K. Bjerklov and T. Jager. Rotation numbers for quasiperiodically forced circle maps-modelocking vs. strict monotonicity. J. Am. Math. Soc., 22(2):353-362, 2009.

[5] N. N. Bogoljubov, J. A. Mitropoliskii, and A. M. Samoılenko. Methods of accelerated convergence in nonlinear mechanics. Hindustan Publishing Corp., Delhi, 1976.

[6] H. W. Broer and M. Levi. Geometrical aspects of stability theory for Hill's equations. Arch. Rational Mech. Anal., 131(3):225-240, 1995.

[7] H. W. Broer, J. Puig, and C. Simó. Resonance tongues and instability pockets in the quasiperiodic Hill-Schrödinger equation. Comm. Math. Phys, 241(2-3):467-503, 2003.

[8] H. W. Broer and C. Simó. Hill's equation with quasi-periodic forcing: resonance tongues, instability pockets and global phenomena. Bol. Soc. Brasil. Mat. (N.S.), 29(2):253-293, 1998.

[9] H. W. Broer and C. Simó. Resonance tongues in Hill's equations: a geometric approach. $J$. Differential Equations, 166(2):290-327, 2000.

[10] V. A. Chulaevsky and Ya. G. Sinal̆. Anderson localization for the 1-D discrete Schrödinger operator with two-frequency potential. Comm. Math. Phys., 125(1):91-112, 1989.

[11] PM Cincotta, CM Giordano, and C. Simó. Phase space structure of multi-dimensional systems by means of the mean exponential growth factor of nearby orbits. Physica D: Nonlinear Phenomena, 182(3-4):151-178, 2003.

[12] N. D. Cong and R. Fabbri. On the spectrum of the one-dimensional Schrödinger operator. Discrete Contin. Dyn. Syst., Ser. B, 9(3-4):541-554, 2008.

[13] C. De Concini and R.A. Johnson. The algebraic-geometric AKNS potentials. Ergodic Theory Dynam. Systems, 7(1):1-24, 1987.

[14] P. Deift and B. Simon. Almost periodic Schrödinger operators III. The absolute continuous spectrum. Comm. Math. Phys., 90:389-341, 1983.

[15] F. Delyon and P. Foulon. Adiabatic invariants and asymptotic behavior of Lyapunov exponents of the Schrödinger equation. J. Statist. Phys., 45(1-2):41-47, 1986.

[16] F. Delyon and P. Foulon. Adiabatic theory, Liapunov exponents, and rotation number for quadratic Hamiltonians. J. Stat. Phys., 49(3-4):829-840, 1987.

[17] E.I. Dinaburg and Y.G. Sinai. The one-dimensional Schrödinger equation with quasi-periodic potential. Funkt. Anal. i. Priloz., 9:8-21, 1975.

[18] L. H. Eliasson. Floquet solutions for the one-dimensional quasi-periodic Schrödinger equation. Comm. Math. Phys., 146:447-482, 1992.

[19] R. Fabbri, R.A. Johnson, and R. Pavani. On the nature of the spectrum of the quasi-periodic Schrödinger operator. Nonlinear Anal. Real World Appl., 3(1):37-59, 2002.

[20] J. Fröhlich, T. Spencer, and P. Wittwer. Localization for a class of one-dimensional quasiperiodic Schrödinger operators. Comm. Math. Phys., 132(1):5-25, 1990.

[21] M. Goldstein and W. Schlag. Fine properties of the integrated density of states and a quantitative separation property of the Dirichlet eigenvalues. Geom. Funct. Anal., 18(3):755-869, 2008 . 
[22] S. Hadj Amor. Hölder continuity of the rotation number for quasi-periodic co-cycles in SL(2,R). Comm. Math. Phys., 287(2):565-588, 2009.

[23] À. Haro and J. Puig. Aubry duality for quasi-periodic potentials i: Lyapunov exponents and cantor spectrum. In preparation.

[24] H.L. He and J. You. Full Measure Reducibility for Generic One-parameter Family of Quasiperiodic Linear Systems. Journal of Dynamics and Differential Equations, 20:1-36, 2008.

[25] M.R. Herman. Une méthode pour minorer les exposants de Lyapunov et quelques exemples montrant le caractère local d'un théorème d'Arnold et de Moser sur le tore de dimension 2. Comment. Math. Helvetici, 58(3), 1983.

[26] S. Jitomirskaya. Ergodic Schrödinger operators (on one foot). Gesztesy, Fritz et al. (ed.), Spectral theory and mathematical physics. A festschrift in honor of Barry Simon's 60th birthday. Ergodic Schrödinger operators, singular spectrum, orthogonal polynomials, and inverse spectral theory. Based on the SimonFest conference, Pasadena, CA, USA, March 2731, 2006. Providence, RI: American Mathematical Society (AMS). Proceedings of Symposia in Pure Mathematics 76, Pt. 2, 613-647, 2007.

[27] R.A. Johnson. Cantor spectrum for the quasi-periodic Schrödinger equation. J. Diff. Eq., 91:88-110, 1991.

[28] R.A. Johnson and J. Moser. The rotation number for almost periodic potentials. Commun. Math. Phys., 84:403-438, 1982

[29] À. Jorba and C. Simó. On the reducibility of linear differential equations with quasiperiodic coefficients. J. Differential Equations, 98(1):111-124, 1992.

[30] À. Jorba and M. Zou. A software package for the numerical integration of ODEs by means of high-order Taylor methods. Experimental Mathematics, 14(1):99-117, 2005.

[31] J.F.C. Kingman. The ergodic theory of subadditive stochastic processes. J. Roy. Statist. Soc. Ser. B, 30:499-510, 1968.

[32] W. Magnus and S. Winkler. Hill's equation. Dover Publications Inc., New York, 1979. Corrected reprint of the 1966 edition.

[33] J. Moser and J. Pöschel. An extension of a result by Dinaburg and Sinai on quasi-periodic potentials. Comment. Math. Helvetici, 59:39-85, 1984.

[34] W.G. Nowak. On simultaneous Diophantine approximation. Rendiconti del Circolo Matematico di Palermo, 33(3):456-460, 1984.

[35] J. Puig. Cantor spectrum and KDS eigenstates. Comm. Math. Phys., 267(3):735-740, 2006.

[36] J. Puig and C. Simó. Analytic families of reducible linear quasi-periodic differential equations. Ergodic Theory Dynam. Systems, 26(2):481-524, 2006.

[37] J. Puig and C. Simó. Resonance tongues and spectral gaps in quasi-periodic Schrödinger operators with one or more frequencies. a numerical exploration. J. Dynam. Differential Equations, 2009. To appear.

[38] R. J. Sacker and G. R. Sell. A spectral theory for linear differential systems. J. Diff. Eq., 27:320-358, 1978 .

[39] C. Simó. Averaging under fast quasiperiodic forcing. In Hamiltonian mechanics (Toruń, 1993), pages 13-34. Plenum, New York, 1994.

[40] C. Simó. Taylor method for the integration of ODE. Lectures given at the Advanced School on Long Time Integrations. Available at http://www.maia.ub.es/dsg/2007/0708simo.ps.gz, 2007.

[41] Ya. G. Sinai. Anderson localization for one-dimensional difference Schrödinger operator with quasiperiodic potential. J. Statist. Phys., 46(5-6):861-909, 1987.

Departament de Matemàtica Aplicada I. Universitat Politècnica de Catalunya. DiaGONAL, 647. 08028 BARCELONA (SPAIN).

E-mail address: joaquim.puig@upc.edu

Departament de Matemàtica Aplicada i AnÀlisi. Universitat de Barcelona. Gran Via de les Corts Catalanes, 585. 08007 Barcelona (Spain).

E-mail address: carles@maia.ub.es 